Bull. Austral. Math. Soc.

VoL. $62(2000) \quad[459-465]$

\title{
QUADRATIC FORMS ASSOCIATED WITH PLANAR ENDOMORPHISMS
}

\author{
G.E. PrInCE
}

Any linear operator $A$ on $\mathbb{E}^{2}$ is shown to have two real quadratic forms on $S^{1}$ associated with it. They represent the expansion and rotation of the map and the eigenvalues of $A$ can be described in a geometrically intrinsic way in terms of the eigenvalues of these two quadratic forms via the formula

$$
\lambda_{1,2}=\frac{1}{2}\left(\lambda_{1}^{\exp }+\lambda_{2}^{\text {exp }}\right) \pm \sqrt{-\lambda_{1}^{\text {rot }} \lambda_{2}^{\text {rot }}} .
$$

A number of theorems concerning these quadratic forms are presented.

\section{INTRODUCTION}

The geometric structure of linear maps is usually analysed through the standard (additive) decomposition into an expansionary part, a shear and a twist through the Euler-Cauchy-Stokes decomposition theorem [3]. However, in the planar case any endomorphism $A$ on $\mathbb{E}^{2}$ has associated with it a pair of quadratic forms $A^{\text {exp }}, A^{\text {rot }}$ on $S^{1}$ which represent the expansion and rotation of the map. In particular, the associated eigenvalues $\lambda_{1}^{\exp }, \lambda_{2}^{\exp }$ and $\lambda_{1}^{\text {rot }}, \lambda_{2}^{\text {rot }}$ are the minimum and maximum values of the expansion and rotation of the map.

The result of the main theorem is that

$$
\lambda_{1,2}=\frac{\lambda_{1}^{\text {exp }}+\lambda_{2}^{\text {exp }}}{2} \pm \sqrt{-\lambda_{1}^{\text {rot }} \lambda_{2}^{\text {rot }}}
$$

where $\lambda_{1,2}$ are the (possibly complex) eigenvalues of $A$.

As a corollary of this result, in the case of a repeated eigenvalue of $A$, the corresponding eigenspace has dimension 2 when $\lambda_{1}^{\text {rot }}=\lambda_{2}^{\text {rot }}=0$ and dimension 1 when only one of these rotations is zero. Apart from their intrinsic attraction and apparent originality, these results find application in the classification of planar flows $[\mathbf{1}, \mathbf{2}]$.

Received 20th March, 2000

The author thanks Grant Cairns, Peter Stacey, Deborah King and Georgia Stathopoulos for their comments.

Copyright Clearance Centre, Inc. Serial-fee code: 0004-9727/00 \$A2.00+0.00. 


\section{QuAdRATIC FORMS ON THE CIRCLE}

Here are some elementary results concerning quadratic forms on $S^{1}$ needed in the next section when the expansion and rotation of a linear operator on $\mathbb{E}^{2}$ are introduced.

THEOREM 2.1. Let $Q: S^{1} \rightarrow \mathbb{R}$ be a non-zero quadratic form on the circle with symmetric matrix representation $[Q]$ (relative to some orthonormal basis) so that

$$
Q(\widehat{u})=[\widehat{u}]^{T}[Q][\widehat{u}]
$$

where $\widehat{u}=(\cos (\phi), \sin (\phi)), \phi \in[0,2 \pi)$. Then the eigenvalues of $Q$ are its maximum and minimum values and these values are achieved in the corresponding eigendirections. The quadratic form is constant if and only if it has a repeated eigenvalue.

ProOF:

$$
\begin{aligned}
\frac{d Q(\widehat{u})}{d \phi} & =\left[\widehat{u}^{\perp}\right]^{T}[Q][\widehat{u}]+[\widehat{u}]^{T}[Q]\left[\widehat{u}^{\perp}\right] \\
& =2\left[\widehat{u}^{\perp}\right]^{T}[Q][\widehat{u}] \\
& =2[\widehat{u}]^{T}[Q]\left[\widehat{u}^{\perp}\right]
\end{aligned}
$$

since $\frac{d}{d \phi}[\widehat{u}]=\left[\widehat{u}^{\perp}\right]$ and $[Q]$ is symmetric. Hence the local extrema occur in the eigendirections of $Q$. Because the domain of $Q$ is $S^{1}$ this pair of local extrema are global in character.

Let $\widehat{u}$ be an eigendirection of $Q$ belonging to $\lambda$, then

$$
Q(\widehat{u})=\lambda[\widehat{u}]^{T}[\widehat{u}]=\lambda .
$$

Clearly if $\lambda$ is a repeated eigenvalue, then $Q$ has this value in every direction.

DEFinition 2.2: The average value of a non-zero quadratic form $Q: S^{1} \rightarrow \mathbb{R}$ is

$$
\bar{Q}:=\frac{1}{2 \pi} \int_{0}^{2 \pi} Q((\cos \phi, \sin \phi)) d \phi .
$$

THEOREM 2.3. Let $Q: S^{1} \rightarrow \mathbb{R}$ be a non-zero quadratic form on $S^{1}$, then

$$
\bar{Q}=\frac{1}{2} \operatorname{trace}(Q) \text {. }
$$

Proof: Suppose that

$$
Q((\cos \phi, \sin \phi))=\alpha \cos ^{2} \phi+2 \beta \cos \phi \sin \phi+\gamma \sin ^{2} \phi
$$

for some $\alpha, \beta, \gamma \in \mathbb{R}$. Then

$$
\begin{aligned}
\bar{Q} & =\frac{1}{2 \pi} \int_{0}^{2 \pi}\left(\alpha \cos ^{2} \phi+2 \beta \cos \phi \sin \phi+\gamma \sin ^{2} \phi\right) d \phi \\
& =\frac{(\alpha+\gamma)}{2}=\frac{1}{2} \operatorname{trace}(Q) .
\end{aligned}
$$


ThEOREM 2.4. Let $Q: S^{1} \rightarrow \mathbb{R}$ be a non-zero quadratic form on $S^{1}$ with average value $\bar{Q}=(1 / 2)$ trace $(Q)$. This average value is achieved in the direction

$$
\widehat{u}_{\mathrm{av}}=\frac{1}{\sqrt{2}}\left(\widehat{u}_{\max }+\widehat{u}_{\min }\right)
$$

where $\widehat{u}_{\max }, \widehat{u}_{\min }$ are the eigendirections corresponding to the $\max / \min$ values of $Q$.

PRoOF: If $Q$ has a repeated eigenvalue the result follows immediately from Theorem 2.1. If $Q$ has a distinct eigenvalues we use $\left\{\widehat{u}_{\max }, \widehat{u}_{\min }\right\}$ as an orthonormal basis for $S^{1}$ and write

$$
\widehat{u}:=\cos \phi \widehat{u}_{\max }+\sin \phi \widehat{u}_{\min }
$$

so that

$$
Q(\widehat{u})=\lambda_{\max } \cos ^{2} \phi+\lambda_{\min } \sin ^{2} \phi
$$

so

and so

$$
Q(\widehat{u})=\frac{1}{2} \operatorname{trace}(Q) \Longleftrightarrow \cos ^{2} \phi=\frac{1}{2}=\sin ^{2} \phi
$$

$$
\widehat{u}_{\mathrm{av}}=\frac{1}{\sqrt{2}}\left(\widehat{u}_{\max }+\widehat{u}_{\min }\right) \text {. }
$$

(This is intended to cover all 4 points on $S^{1}$.)

\section{EIGENVALUES OF PLANAR ENDOMORPHISMS}

Let $A$ be a linear operator on $\mathbb{E}^{2}$, then for any non-zero $u \in \mathbb{E}^{2}$ with $\widehat{u}:=u /\|u\|$

$$
A(u)=(A(u) \cdot \widehat{u}) \widehat{u}+\left(A(u) \cdot \widehat{u}^{\perp}\right) \widehat{u}^{\perp},
$$

where $u^{\perp}:=R_{\pi / 2}(u)$ (so that if $u=\left(u^{1}, u^{2}\right)$ then $u^{\perp}=\left(-u^{2}, u^{1}\right)$ ). Hence

$$
A(u)=(A(\widehat{u}) \cdot \widehat{u}) u+\left(A(\widehat{u}) \cdot \widehat{u}^{\perp}\right) u^{\perp}
$$

and, since the adjoint of $R_{\pi / 2}$ is $R_{-\pi / 2}$ relative to the standard inner product,

$$
A(u)=(A(\widehat{u}) \cdot \widehat{u}) u+\left(R_{-\pi / 2} \circ A(\widehat{u}) \cdot \widehat{u}\right) u^{\perp} .
$$

If the matrix representation of $A$ (relative to the natural basis) is

$$
[A]=\left(\begin{array}{ll}
a_{11} & a_{12} \\
a_{21} & a_{22}
\end{array}\right)
$$

then the matrix representations of the two quadratic forms

$$
A^{\exp }(\widehat{u}):=A(\widehat{u}) \cdot \widehat{u}, \quad A^{\text {rot }}(\widehat{u}):=R_{-\pi /} \circ A(\widehat{u}) \cdot \widehat{u}
$$

are

$$
\left[A^{\exp }\right]=\left(\begin{array}{cc}
a_{11} & \frac{1}{2}\left(a_{12}+a_{21}\right) \\
\frac{1}{2}\left(a_{12}+a_{21}\right) & a_{22}
\end{array}\right),\left[A^{\text {rot }]}=\left(\begin{array}{cc}
a_{21} & \frac{1}{2}\left(a_{22}-a_{11}\right) \\
\frac{1}{2}\left(a_{22}-a_{11}\right) & -a_{12}
\end{array}\right) .\right.
$$


Definition 3.1: $A^{\exp }(\widehat{u})$ and $A^{\text {rot }}(\widehat{u})$ are respectively called the expansion and rotation of the operator $A$ in the direction of $\widehat{u}$. The average values of the expansion and rotation of $A$ are the average values of $A^{\exp }(\widehat{u})$ and $A^{\text {rot }}(\widehat{u})$ respectively.

It is interesting to compare this result with the standard Euler-Cauchy-Stokes decomposition theorem [3]:

TheOREM 3.2. (Euler-Cauchy-Stokes Decomposition Theorem) The matrix representation of $A$ can be uniquely decomposed as

$$
[A]=\frac{\Theta}{2} I_{2}+\Sigma+\Omega
$$

where $\Theta:=$ trace $([A]$ ) (so that $\Theta / 2$ is the average expansion of $A$ ), $\Sigma$ is a trace-free symmetric matrix representing shear and $\Omega$ is a skew-symmetric matrix representing the twist of the map. Explicitly

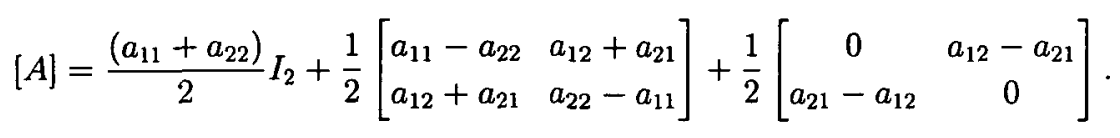

It is a simple matter to show (in an obvious abuse of notation) that

$$
A^{\exp }(\widehat{u})=\overline{A^{\exp }}+\Sigma(\widehat{u}) \cdot \widehat{u}, \quad A^{\text {rot }}(\widehat{u})=\overline{A^{\text {rot }}}+\Sigma(\widehat{u}) \cdot \widehat{u}^{\perp} .
$$

Now we apply Theorem 2.1 to the rotation and expansion of $A$. Part (c) of the following theorem follows from the definition of $A^{\text {rot }}$.)

PROPOSITION 3.3.

(a) If the quadratic form $A^{\exp }$ is not zero, then its eigenvalues are the maximum and minimum values of the expansion of the map $A$ and these are achieved in the corresponding eigendirections. The map $A$ has constant expansion in all directions if and only if $A^{\exp }$ has a repeated eigenvalue.

(b) If the quadratic form $A^{\text {rot }}$ is not zero then its eigenvalues are the maximum and minimum values of the rotation of the map $A$ and these are achieved in the corresponding eigendirections. The map $A$ has constant rotation in every direction if and only if $A^{\text {rot }}$ has a repeated eigenvalue.

(c) The zeros of $A^{\text {rot }}$ occur in the eigendirections of $A$.

The main theorem relates the eigenvalues of $A$ to those of $A^{\exp }$ and $A^{\text {rot: }}$

THEOREM 3.4. Let the (possibly repeated) eigenvalues of $A$ be $\lambda_{1}, \lambda_{2}$ and those of $A^{\exp }$ and $A^{\text {rot }}$ be $\lambda_{1}^{\exp }, \lambda_{2}^{\exp }$ and $\lambda_{1}^{\text {rot }}, \lambda_{2}^{\text {rot }}$ respectively. Then

$$
\lambda_{1,2}=\frac{\lambda_{1}^{\exp }+\lambda_{2}^{\exp }}{2} \pm \sqrt{-\lambda_{1}^{\text {rot }} \lambda_{2}^{\text {rot }}}
$$


PrOOF: This is a straight forward calculation using the matrix representations of $A, A^{\text {exp }}$ and $A^{\text {rot }}$ (relative to the natural basis). The crucial facts are:

$$
\operatorname{det}\left[A^{\mathrm{rot}}\right]=-\frac{1}{4}(\operatorname{trace}[A])^{2}+\operatorname{det}[A]
$$

and

$$
\operatorname{trace}\left[A^{\text {exp }}\right]=\operatorname{trace}[A]
$$

This theorem shows that the average expansion of $A$ is the arithmetic mean of the maximum and minimum expansions. More strikingly it shows that $A$ has complex eigenvalues precisely when the maximum and minimum rotations have the same sign, so that the map rotates every direction in the same sense. This is intuitively satisfying given the behaviour of pure planar rotations. Finally, the theorem has a corollary which explains the geometric multiplicity of repeated eigenvalues of the map $A$.

COROLlary 3.5. If the linear operator $A$ has a repeated eigenvalue $\lambda$ its geometric multiplicity is 2 if and only if $\lambda_{1}^{\text {rot }}=0=\lambda_{2}^{\text {rot }}$ (so that $A^{\text {rot }}$ is the zero quadratic form) and its geometric multiplicity is 1 if and only if only one of $\lambda_{1}^{\text {rot }}, \lambda_{2}^{\text {rot }}$ is zero.

Proof: The main theorem indicates that the repetition of $\lambda$ is equivalent to $\lambda_{1}^{\text {rot }} \cdot \lambda_{2}^{\text {rot }}=0$. Notice also that $A^{\text {rot }}(\widehat{u})=0$ in any eigendirection of $A$ (whether the corresponding eigenvalue is repeated or not).

Now suppose that $\lambda$ has geometric multiplicity 2 so that $A=\lambda I$. Then $A^{\text {rot }}=0$ so that $\lambda_{1}^{\text {rot }}, \lambda_{2}^{\text {rot }}$ are trivially zero. On the other hand, if $\lambda_{1}^{\text {rot }}=0=\lambda_{2}^{\text {rot }}$ then $A^{\text {rot }}=0$ since $\lambda_{1}^{\text {rot }}, \lambda_{2}^{\text {rot }}$ are the global maximimum and minimum values of the rotation of $A$. Thus

$$
A(u)=A^{\exp }(\widehat{u}) u, \quad \forall u \in \mathbb{E}^{2}
$$

and so the repeated eigenvalue has geometric multiplicity 2 .

It is an immediate consequence of this argument that geometric multiplicity 1 is equivalent to only one of $\lambda_{1}^{\text {rot }}, \lambda_{2}^{\text {rot }}$ being zero.

This corollary shows that a repeated eigenvalue occurs when a map's rotation is zero in at least one direction (and the eigenvalue achieves geometric multiplicity 2 only when the map itself is a pure expansion).

We can now classify the eigenvalues of linear operators on $\mathbb{E}^{2}$ according to their corresponding rotations using $\lambda_{1}^{\text {rot }} \leqslant A^{\text {rot }}(\widehat{u}) \leqslant \lambda_{2}^{\text {rot }}, \forall \widehat{u} \in S^{1}$ :

\begin{tabular}{c|l} 
zeros of $A^{\text {rot }}$ on $[0, \pi)$ & Eigenvalues of $A$ \\
\hline none & complex \\
1 & repeated, geometric multiplicity 1 \\
2 & real and distinct \\
$\infty$ & repeated, geometric multiplicity 2
\end{tabular}


Needless to say the eigenvectors of $A$ are the zeros of $A^{\text {rot }}$.

Finally, two remarks: while the constructions of $A^{\text {rot }}$ and $A^{\exp }$ are not invariant under similarity transformations, their determinant and trace respectively are. That is, the determinant of the symmetrisation of $P^{-1} \circ\left(R_{-\pi / 2} \circ A\right) \circ P$ is the same as that of the symmetrisation of $R_{-\pi / 2} \circ A$ and similarly for the trace of the symmetrisation of $P^{-1} \circ A \circ P$. The second remark is that any inner product for $\mathbb{R}^{2}$ would have done: the rotation $R_{-\pi / 2}$ being replaced by a linear map $R$ with $R^{2}(u)=-u$ and $R(u)$ orthogonal to $u$ (for nonzero $u$ ).

\section{Generalisations}

There are two clear directions for generalisation of these results: one is to $\mathbb{C}^{2}$ and the other is to $\mathbb{E}^{n}$. The $\mathbb{C}^{2}$ results are rather disappointing inasmuch as the two quadratic forms $A^{\text {exp }}, A^{\text {rot }}$ of the real case do not become Hermitian quadratic. If $u=\left(u^{1}, u^{2}\right)$, then $u^{\perp}=\left(-\bar{u}^{2}, \bar{u}^{1}\right)$ and the map corresponding to $R_{\pi / 2}$ is then antilinear. If we denote this map by $\bar{R}_{\pi / 2}$ we have

$$
A(u)=(A(\widehat{u}) \cdot \widehat{u}) u+\left(\bar{R}_{-\pi / 2} \circ A(\widehat{u}) \cdot \widehat{u}\right) u^{\perp}
$$

where $\bar{R}_{-\pi / 2} \circ A: \mathbb{C}^{2} \rightarrow \mathbb{C}^{2}$ is antilinear. The quadratic forms $A^{\text {exp }}, A^{\text {rot }}$ defined by

$$
A^{\exp }(\widehat{u})=A(\widehat{u}) \cdot \widehat{u}, A^{\text {rot }}(\widehat{u})=\bar{R}_{-\pi / 2} \circ A(\widehat{u}) \cdot \widehat{u}
$$

arise from sesquilinear and completely antilinear maps on $\mathbb{C}^{2} \times \mathbb{C}^{2}$ respectively. Neither quadratic form has an a priori Hermitian matrix representation although $A^{\text {rot }}$ has a symmetric matrix representation. However, the result of the main theorem still holds for the eigenvalues of the 3 matrices

$$
\left(\begin{array}{ll}
a_{11} & a_{12} \\
a_{21} & a_{22}
\end{array}\right), \quad\left(\begin{array}{cc}
a_{11} & \frac{1}{2}\left(a_{12}+a_{21}\right) \\
\frac{1}{2}\left(a_{12}+a_{21}\right) & a_{22}
\end{array}\right), \quad\left(\begin{array}{cc}
a_{21} & \frac{1}{2}\left(a_{22}-a_{11}\right) \\
\frac{1}{2}\left(a_{22}-a_{11}\right) & -a_{12}
\end{array}\right) .
$$

The corollary to the main theorem also holds because a repeated eigenvalue of $A$ means that both eigenvalues of the third matrix above must be real with at least one zero.

The generalisation to $\mathbb{E}^{n}$ seems straight forward in one direction at least. If a linear operator on $\mathbb{E}^{n}$ has an invariant 2-space, the behaviour of its restriction to this 2-space can be analysed with the results given here. This is probably more interesting when the 2 -space is not a direct sum of eigenspaces. The case of orthogonal operators on $\mathbb{E}^{n}$ is probably the simplest.

On the other hand on $\mathbb{E}^{n}$ one might try and generalise the decomposition of $A(u)$ into components along $\widehat{u}$ and along its orthogonal complement: 


$$
\begin{aligned}
A(u) & =(A(u) \cdot \widehat{u}) \widehat{u}+\sum_{i=1}^{n-1}\left(A(u) \cdot \widehat{u}_{i}^{\perp}\right) \widehat{u}_{i}^{\perp}, \\
& =(A(\widehat{u}) \cdot \widehat{u}) u+\sum_{i=1}^{n-1}\left(A(\widehat{u}) \cdot R_{i}(\widehat{u})\right) u_{i}^{\perp},
\end{aligned}
$$

where $\left\{u_{1}^{\perp}, \ldots, u_{n-1}^{\perp}\right\}$ is any orthogonal basis for the orthogonal complement of $\operatorname{Sp}\{u\}$ with all elements having the same length as $u$. The rotations $R_{i}$ are the linear operators on $\mathbb{E}^{n}$ satisfying

$$
R_{i}(u)=u_{i}^{\perp}, R_{i}\left(u_{i}^{\perp}\right)=-u(\text { no sum }), R_{i}\left(u_{j}^{\perp}\right)=u_{j}^{\perp}, j \neq i .
$$

However, these rotations in general depend on $u$ unlike the $\mathbb{E}^{2}$ case. This is easily seen in $\mathbb{E}^{3}$ where each of the $R_{i}$ has exactly one real eigenspace spanned by the appropriate $u_{j}^{\perp}$. So it seems that analysis in the $\mathbb{E}^{n}$ case is best accomplished by examination of invariant two spaces.

\section{REFERENCES}

[1] G. Prince and G. Stathopoulos, 'The geometry of planar flows', (preprint, Department of Mathematics, La Trobe University, 1999). Available at http://www . latrobe.edu.au/ww/mathstats/Staff/prince.html.

[2] G. Stathopoulos, Geometry of planar flows, (M.Sc. Thesis) (Department of Mathematics, La Trobe University, 1998).

[3] C. Truesdell, $A$ first course in rational continuum mechonics, Pure and Applied Mathematics 71 (Academic Press, Boston, MA, 1977).

Department of Mathematics

La Trobe University

Bundoora, Vic. 3083

Australia.

e-mail: G.Prince@latrobe.edu.au 